\title{
Secondary stent-in-stent self-expanding metallic stent placement for early stent occlusion due to mucosal oedema in the gastrojejunal anastomosis after palliative subtotal gastrectomy for locally advanced gastric cancer
}

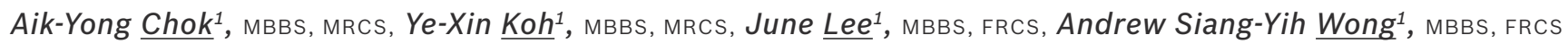

\begin{abstract}
Herein, we report a case of early in-stent occlusion due to the ingrowth of soft oedematous mucosal tissue through the lattices of an uncovered stent, which was used for palliation of a postoperative gastrojejunal anastomotic stricture. The in-stent occulsion was treated with the deployment of a second stent, which was covered, within the first stent. This led to successful resolution of the occlusion.
\end{abstract}

Keywords: secondary stent-in-stent placement, SEMS

\section{INTRODUCTION}

The endoscopic deployment of self-expanding metallic stents (SEMS) for palliation of malignant gastric outlet obstruction (GOO) was first described by Truong et al in 1992.(1) Since then, numerous studies have demonstrated the advantages and efficacy of this palliative treatment option, which has relatively high success rates and low procedure-associated morbidity and mortality. ${ }^{(2)}$

In the presence of an inoperable disease, enteral stenting is a simple yet safe treatment option to relieve obstructive symptoms and enable oral nutrition. However, common complications include stent obstruction and migration. Stent obstruction was found to be largely secondary to malignant tumour ingrowth, ${ }^{(2)}$ while other causes include malfunction, fracture, and even large impacted food boluses. ${ }^{(3)}$ In such cases, therapeutic options include: (a) the removal of the occluded stent and redeployment of another in its place; (b) the coaxial placement of a second stent within the first stent, also described as secondary stent-in-stent SEMS placement; and (c) palliative surgical bypass. ${ }^{(4)}$

Herein, we report a case of early in-stent occlusion due to ingrowth of soft oedematous mucosal tissue through the lattices of an uncovered stent, which was used for palliation of a postoperative gastrojejunal anastomotic stricture. This was subsequently successfully treated using secondary stent-in-stent SEMS placement.

\section{CASE REPORT}

A 62-year-old man underwent a palliative subtotal gastrectomy with Bilroth II gastrojejunostomy for a locally advanced gastric adenocarcinoma, classified as signet ring cell type using World Health Organization's histologic classification of gastric tumours. ${ }^{(5)}$ Histopathology revealed a stage T4aN3a tumour that was poorly differentiated and diffuse (based on Lauren classification $\left.^{(6)}\right)$, with 9 of 25 lymph nodes involved, and a positive distal resection margin.

Three months after the gastrectomy, the patient presented with signs and symptoms of intestinal obstruction that had persisted for a period of three days. Computed tomography imaging of the abdomen and pelvis revealed a closed-loop large bowel obstruction due to locally recurrent disease involving the hepatic flexure and transverse colon, with resultant dilated caecum and ascending colon. The patient underwent laparotomy and colocolic bypass. Multiple small peritoneal nodules were seen intraoperatively, suggesting disseminated intraperitoneal disease.

Postoperative recovery was complicated by persistent signs and symptoms of GOO, including vomiting and positive succussion splash, prompting a water-soluble contrast meal study, which was performed on the postoperative Day 8. The study revealed no immediate transit of contrast beyond the anastomosis into the efferent jejunal limb. After 16 mins and with the aid of dynamic manoeuvres, contrast was seen trickling intermittently downstream, forming a smooth rat's tail appearance (Fig. 1).

The patient subsequently underwent a gastroscopy, which established the presence of an occlusion in the efferent limb of the gastrojejunal anastomosis. A guidewire was passed through the stenosed segment, and a 9-cm length, 2.2-cm diameter WallFlex ${ }^{\circledR}$ uncovered duodenal stent (Boston Scientific Co, Natick, MA, USA) was deployed across the segment. Symptomatic

${ }^{1}$ Department of General Surgery, Changi General Hospital, Singapore

Correspondence: Dr Chok Aik-Yong, Resident, Department of General Surgery, Changi General Hospital, 2 Simei Street 3, Singapore 529889. chokaikyong@gmail.com 


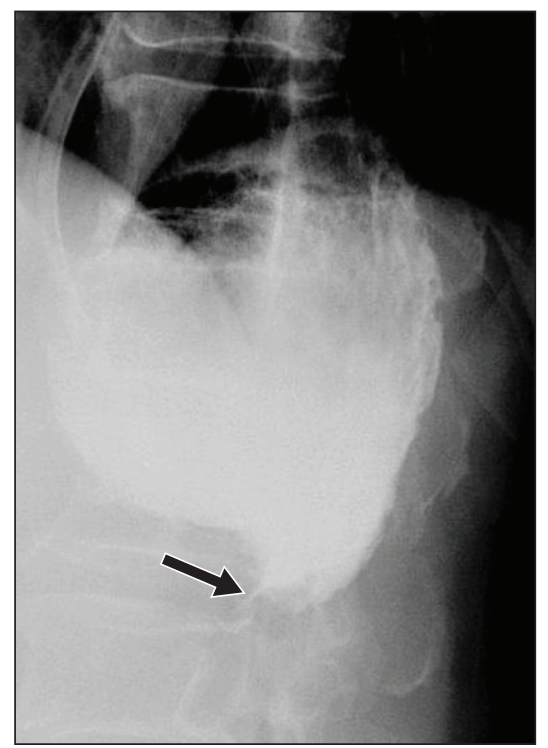

Fig. 1 Radiographic image during the water-soluble contrast meal shows no immediate transit of contrast beyond the gastrojejunal anastomosis. The arrow shows the smooth rat's tail appearance that was seen after dynamic manoeuvres.

improvement was reported after the procedure, and the patient was progressed to feed and a finely minced diet. However, he experienced recurrent symptoms of GOO four days later. Abdominal radiography showed a well-placed enteral stent, with no evidence of stent migration or stenosis (Fig. 2).

Hence, a repeat gastroscopy was performed, with the possibility of argon plasma coagulation of the ingrowing tumour tissue that may be causing the in-stent occlusion. Although the stent was observed to still be in good position, oedematous small bowel mucosa was seen passing through the stent lattices, causing in-stent obstruction (Fig. 3a). The scope could be easily passed beyond the obstruction with minimal resistance (Fig. 3b). The oedematous mucosa ingrowth was soft in consistency. As the mucosa ingrowth was likely benign based on its clinical appearance and soft consistency, no biopsy was performed. In addition, as it was not a hard tumour ingrowth, argon plasma coagulation was not indicated in this clinical setting.

A stent-in-stent procedure was performed using a 100-mm Niti-S ${ }^{\text {TM }}$ covered enteral colonic stent (Taewoong Medical Co Ltd, Seoul, South Korea) to bridge the obstruction (Figs. 4 \& 5). The patient had no further episodes of vomiting after the procedure. He was uneventfully progressed to a blended diet, and was discharged well three days after the stent-in-stent procedure. He has since been started on palliative chemotherapy, and the double stents have remained clinically patent for more than eight weeks.

\section{DISCUSSION}

Malignant GOO is a late complication of gastric, duodenal, pancreatic and biliary malignancies. Therapeutic options include surgical bypass, and more recently, the use of SEMS. A meta-analysis on endoscopic and surgical procedures for GOO palliation showed that endoscopic stenting had

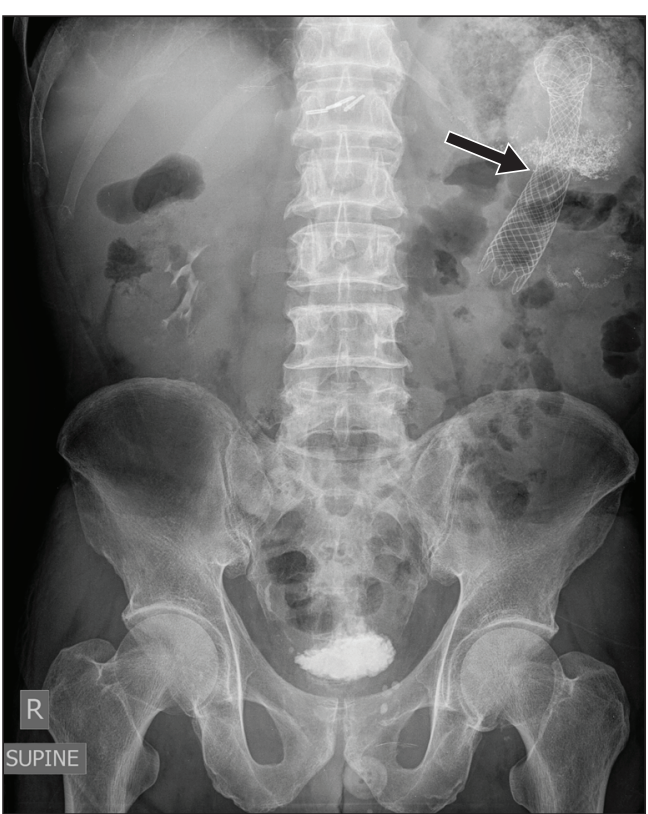

Fig. 2 Radiographic image of the abdomen shows the deployed gastrojejunal stent in place (arrow).

better outcomes in terms of clinical success, and reduction in procedure-related morbidity, mortality and length of hospital stay. ${ }^{(7)}$ The placement of SEMS in the palliation of a recurrent malignant obstruction (due to anastomotic stricture or local tumour recurrence after gastric surgery) has been shown to be technically feasible and clinically effective. ${ }^{(8)}$

Although there have been concerns about the clinical use of SEMS in peritoneal carcinomatosis due to the risk of multifocal obstruction, ${ }^{(9)}$ a study by Mendelsohn et $\mathrm{al}^{(10)}$ showed similar clinical outcomes in the group with and without carcinomatosis (clinical success rates of $81 \%$ and $84 \%$, respectively). In the present case, we opted for SEMS even though our patient had peritoneal carcinomatosis, as the obstruction was identified to be at the gastrojejunal site, with the other obstruction in the large bowel already bypassed.

In several prospective studies, the stent patency time, failure rate and reintervention rate of both covered and uncovered stents were found to be comparable. ${ }^{(11)}$ Although uncovered stents are favoured, mainly due to the low risk of dislodgement and migration, ${ }^{(12)}$ local tissue hyperplasia as well as ingrowth of tumour and oedematous tissue through the fenestrations of uncovered stents can lead to stent failure. Covered stents, however, are associated with a slightly higher incidence of migration after deployment.

In the present case, a WallFlex ${ }^{\circledR}$ uncovered duodenal stent was deployed first. This stent is made of nitinol, an alloy of nickel and titanium. Nitinol has increased flexibility, which is helpful for stenting sharply angulated regions, but this is at the cost of a lesser radial force, relative to stents made with other metals. ${ }^{(13)}$ The decision to first use an uncovered stent was due to several reasons. Firstly, most commonly available pyloric or enteral stents are uncovered. Also, a stent deployed at such a location (i.e. at the efferent limb of the gastrojejunal 

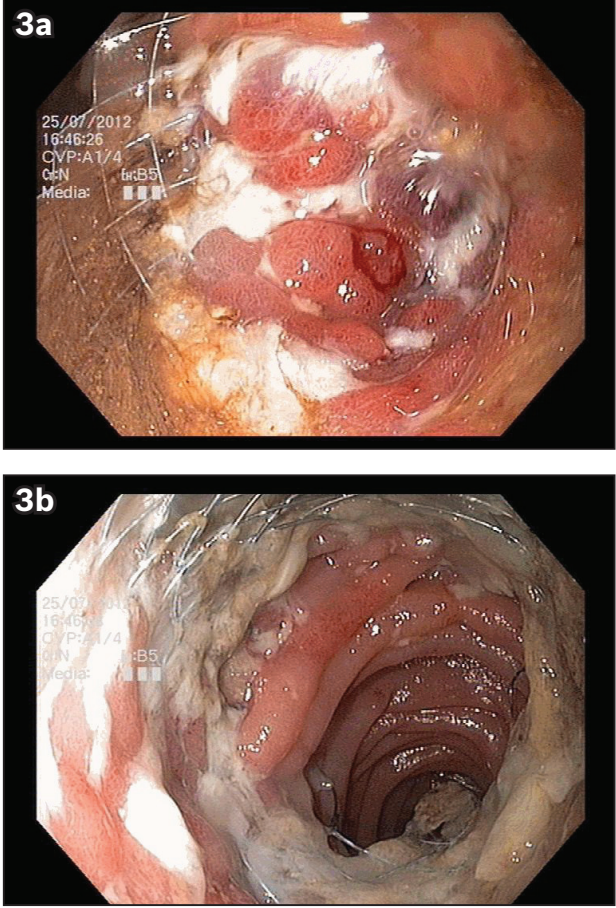

Fig. 3 Endoscopic images shows (a) the direct frontal scope view of the oedematous mucosa causing the in-stent occlusion; and (b) the passage of the scope through the oedematous mucosa with minimal resistance.

anastomosis) would be more susceptible to migration as only one end of the stent has anchorage on a luminal wall. This is in contrast with stents placed in other locations such as the mid-oesophagus, where both ends of the stent are in contact with the luminal wall. Finally, unlike the present case, malignant in-stent tumour ingrowth usually takes some time to occur and can be easily treated with argon plasma coagulation ablation. In other words, the short-term patency rate of an uncovered stent was expected to be similar to that of a covered stent. In the present case, the failure of the uncovered stent was found to be due to the presence of reactive tissue oedema and mucosal ingrowth that caused in-stent occlusion. With the subsequent deployment of a Niti-S ${ }^{T M}$ covered enteral colonic stent within the uncovered stent, the combined radial force of the two nitinol stents and the nature of the covered stent ensured in-stent patency by extruding the soft mucosal oedema that had encroached the stent lumen via the lattices of the uncovered stent.

It is reported that $18 \%-22 \%$ of patients who undergo SEMS placement for management of malignant GOO experience recurrent obstruction (caused by progressive tumour growth or stent migration) that necessitates additional interventions. ${ }^{(14)}$ Current therapeutic approaches include not only palliative bypass surgery, but also the insertion of a second stent inside the previous stent, the so-called stent-in-stent SEMS placement. ${ }^{(15)}$ Park et al ${ }^{(4)}$ reported a clinical success of $88.3 \%(68 / 77)$ in relieving GOO with stent-in-stent placement. Although there was stent malfunction in 26 of the 68 patients who underwent stent-in-stent placement, these malfunctions were successfully managed with additional SEMS placement

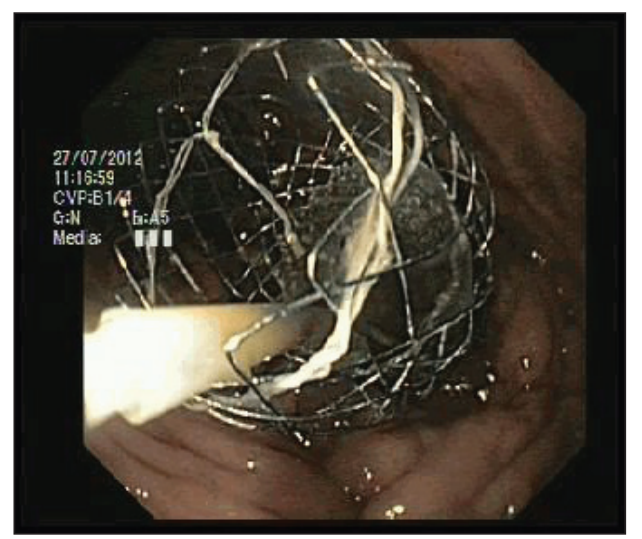

Fig. 4 Endoscopic image shows deployment of a $100-\mathrm{mm}$ Niti-S ${ }^{\mathrm{TM}}$ covered enteral colonic stent within the previous uncovered stent.

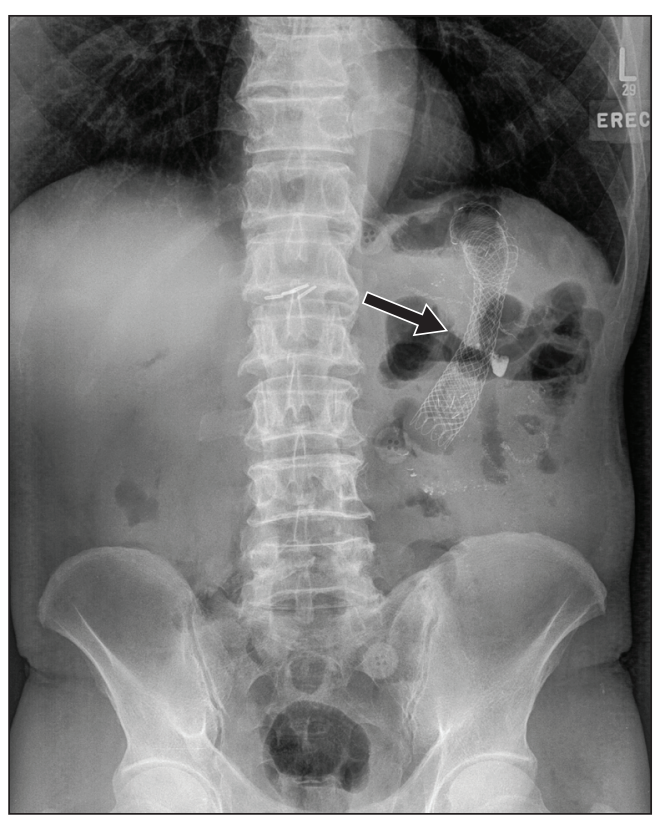

Fig. 5 Radiographic image of the abdomen shows the stent-in-stent deployed across the gastrojejunal anastomosis (arrow).

and balloon dilatation. Ultimately, only two patients of the 26 required surgical bypass. ${ }^{(4)}$ This reflected the use of stent-instent placement as a simple and yet effective method of palliation. Even if failure occurred, it was manageable with endoscopic methods.

To the best of our knowledge, there have been no reports of ingrowth of oedematous mucosal tissue resulting in early occlusion of an uncovered enteral stent and recurrent GOO at the gastrojejunal anastomosis after palliative gastrectomy in the same individual. Although there is no clear evidence in the literature to support the management of such a case using parenteral and intravenous steroids and anti-inflammatory medications (to reduce tissue oedema), this was one of the conservative measures considered in the present case. However, the signs and symptoms of GOO in our patient persisted despite the use of nonsteroidal anti-inflammatory drugs for five days. Furthermore, as the albumin level in our patient was $30 \mathrm{~g} / \mathrm{L}$ at the time of presentation, it was perceived that the use of albumin infusion would not significantly alleviate 
the obstruction caused by the oedematous mucosal tissue, and thus would be of little benefit to the patient.

The present case study highlights the technical feasibility and clinical effectiveness of deploying a second enteral stent within the first stent. This procedure makes possible the option of combining the advantages of both uncovered and covered enteral stents in the treatment of recurrent malignant GOO. In fact, combined double-layered stents with an outer uncovered layer and an inner covered layer, have been designed and implemented. Evaluation of the long-term performance and outcome of double stent placement will require larger case series and clinical trials. ${ }^{(16,17)}$ Since various types of enteral stents with diverse functional characteristics are available to the physician, the choice of stents for placement should be tailored to each patient.

\section{REFERENCES}

1. Truong S, Bohndorf V, Geller H, Schumpèlick V, Günther RW. Selfexpanding metal stents for palliation of malignant gastric outlet obstruction. Endoscopy 1992; 24:433-5.

2. Dormann A, Meisner S, Verin N, Wenk Lang A. Self-expanding metal stents for gastroduodenal malignancies: systematic review of their clinical effectiveness. Endoscopy 2004; 36:543-50.

3. Phillips MS, Gosain S, Bonatti $\mathrm{H}$, et al. Enteral stents for malignancy: a report of 46 consecutive cases over 10 years, with critical review of complications. J Gastrointest Surg 2008; 12:2045-50.

4. Park JC, Park JJ, Cheoi K, et al. Clinical outcomes of secondary stent-instent self-expanding metal stent placement for primary stent malfunction in malignant gastric outlet obstruction. Dig Liver Dis 2012, 44:999-1005.

5. Jass JR, Sobin LH, Watanabe H. The World Health Organization's histologic classification of gastrointestinal tumors. A commentary on the second edition. Cancer 1990; 66:2162-7.
6. Lauren P. The two histological main types of gastric carcinoma: diffuse and so-called intestinal-type carcinoma. An attempt at a histo-clinical classification. Acta Pathol Microbiol Scand 1965; 64:31-49.

7. Hosono S, Ohtani H, Arimoto $\mathrm{Y}$, Kanamiya Y. Endoscopic stenting versus surgical gastroenterostomy for palliation of malignant gastroduodenal obstruction: a meta-analysis. J Gastroenterol 2007; 42:283-90.

8. Kim HJ, Park JY, Bang S, et al. Self-expandable metal stents for recurrent malignant obstruction after gastric surgery. Hepatogastroenterology 2009; 56:914-7.

9. Baron TH, Harewood GC. Enteral self-expandable stents. Gastrointest Endosc 2003; 58:421-33.

10. Mendelsohn RB, Gerdes H, Markowitz AJ, DiMaio CJ, Schattner MA. Carcinomatosis is not a contraindication to enteral stenting in selected patients with malignant gastric outlet obstruction. Gastrointest Endosc 2011; 73:1135-40.

11. Kim CG, Choi IJ, Lee JY, et al. Covered versus uncovered self-expandable metallic stents for palliation of malignant pyloric obstruction in gastric cancer patients: a randomized, prospective study. Gastrointest Endosc 2010; 72:25-32.

12. Maetani I, Ukita T, Tada T, et al. Metallic stents for gastric outlet obstruction: reintervention rate is lower with uncovered versus covered stents, despite similar outcomes. Gastrointest Endosc 2009; 69:806-12

13. Varadarajulu S, Banerjee S, Barth B, et al. Enteral stents. Gastrointest Endosc 2011; 74:455-64

14. Jeurnink SM, van Eijck CH, Steyerberg EW, Kuipers EJ, Siersema PD. Stent versus gastrojejunostomy for the palliation of gastric outlet obstruction: a systematic review. BMC Gastroenterology 2007; 7:18.

15. Homs MY, Steyerberg EW, Kuipers EJ, et al. Causes and treatment of recurrent dysphagia after self-expanding metal stent placement for palliation of esophageal carcinoma. Endoscopy 2004; 36:880-6.

16. Song GA, Kang DH, Kim TO, et al. Endoscopic stenting in patients with recurrent malignant obstruction after gastric surgery: uncovered versus simultaneously deployed uncovered and covered (double) self-expandable metal stents. Gastrointest Endosc 2007; 65:782-7.

17. Lee SM, Kang DH, Kim GH, et al. Self-expanding metallic stents for gastric outlet obstruction resulting from stomach cancer: a preliminary study with a newly designed double-layered pyloric stent. Gastrointest Endosc 2007; 66:1206-10 